\title{
Does a thoracoscopic approach provide better outcomes for pulmonary metastases?
}

\author{
Aizemaiti Rusidanmu ${ }^{1}$, Wenjie Chin ${ }^{2}$, Jinming Xu ${ }^{1}$, Luming Wang ${ }^{1}$, Zhehao ${ }^{1}{ }^{1}$, Xiayi Lv $^{1}$, Jian $\mathrm{Hu}^{1}$ \\ ${ }^{1}$ Department of Thoracic Surgery, The First Affiliated Hospital, Zhejiang University School of Medicine, Hangzhou, China; ${ }^{2}$ Division of \\ Hepatobiliary and Pancreatic Surgery, Department of Surgery, The First Affiliated Hospital, Zhejiang University School of Medicine, Hangzhou, \\ China \\ Contributions: (I) Conception and design: J Hu; (II) Administrative support: None; (III) Provision of study materials or patients: None; (IV) Collection \\ and assembly of data: A Rusidanmu, W Chin, L Wang; (V) Data analysis and interpretation: J Xu, Z He, X Lv; (VI) Manuscript writing: All authors; \\ (VII) Final approval of manuscript: All authors. \\ Correspondence to: Jian Hu. Department of Thoracic Surgery, The First Affiliated Hospital of Zhejiang University, 79 Qingchun Road, Hangzhou, \\ China. Email: dr_hujian@zju.edu.cn.
}

\begin{abstract}
Traditional open thoracotomy (OT) is the gold standard treatment for patients with pulmonary metastases. However, it remains controversial whether video-assisted thoracic surgery (VATS) can provide comparable outcomes to OT. We conducted this review to compare the outcomes of VATS with OT in pulmonary metastasectomy (PM). Relevant studies published up to November 2019 were identified from PubMed data base and screened. Studies were then selected by the researchers based on our selection criteria. Data including the type of study, patient groups, outcomes and key results were extracted from the included studies and summarized. Screening of 2,788 papers identified 9 that were relevant to our research question. The authors, dates of publication, journal details, type of study, patient groups, outcomes and key results from these papers were summarized. All 9 studies documented the survival rate (1-, 3- and 5-year survival). Metastases from colorectal cancer were investigated in three studies, and metastases from sarcoma were investigated in one study. The overall survival rate of VATS was not inferior to that of OT in patients with pulmonary metastases. VATS was also associated with better perioperative results compared with OT. In conclusion, VATS is suitable as an alternative surgical technique for PM.
\end{abstract}

Keywords: Lung metastasectomy; pulmonary metastases; video-assisted thoracic surgery (VATS); video-assisted thoracoscopic surgery; open thoracotomy (OT)

Submitted Jan 13, 2020. Accepted for publication Jul 03, 2020.

doi: $10.21037 /$ jtd-19-3958

View this article at: http://dx.doi.org/10.21037/jtd-19-3958

\section{Introduction}

Cancer frequently metastasizes in the lungs (1). As pulmonary metastases were traditionally viewed as systemic dissemination, surgical treatment was reserved only for palliation purposes. It is now recognized that pulmonary metastasectomy (PM) plays an important role in treating a variety of tumors (2). Indeed, for patients with limited pulmonary metastases, surgical resection can improve the clinical outcomes (3). Currently, PM can be achieved using either traditional open thoracotomy (OT) or video-assisted thoracoscopic surgery (VATS). Recent developments in endoscopic devices and surgical expertise have dramatically expanded the effectiveness of VATS in thoracic surgery. Compared with traditional OT, VATS is more effective in lung parenchyma preservation and pain control (4). Moreover, increasing evidence suggests that VATS is associated with improved outcomes in patients requiring additional surgical resections due to recurrence (5-7). However, as VATS resection is unlikely to reveal radiologically undetected parenchymal lesions due to restricted bimanual palpation at the portholes $(8,9)$, the 
long-term consequences in patients with pulmonary metastases remain controversial. In this study, we reviewed and compared the operative strategies and outcomes of PM documented in previous studies, with a focus on patient evaluations, prognostic factors, and surgical strategies. We present the following article in accordance with the Narrative Review reporting checklist (available at http:// dx.doi.org/10.21037/jtd-19-3958).

\section{Eligibility criteria}

We defined the eligibility criteria to include studies that (I) were published in English; (II) compared patients treated using VATS and patients treated using conventional OT; and (III) evaluated oncological outcomes by comparison with the baseline values. Studies of adult patients who were diagnosed with pulmonary metastases and underwent metastasectomy were included based on the American College of Radiology ACR Appropriateness Criteria for screening for pulmonary metastatic disease. Studies based on animal models or in vitro cultures were excluded.

\section{Search strategy}

Two investigators (AR and WC) conducted a literature search in PubMed for studies published up to November 2019 using the following search terms: ('lung metastases' OR 'pulmonary metastases') AND ('VATS' OR 'minimally invasive surgery' OR 'thoracoscopic') AND ('surgery' OR 'surgical management'). We limited our search to studies that involved human adults. The reference lists of the selected studies were used to cross-reference and search for additional relevant articles.

\section{Data extraction and analysis}

We scored the quality (study randomization, double blinding, withdrawals and dropouts) of the articles using the Jadad criteria (10). Studies scoring below 2 were considered poor, and those scoring between 3 and 5 were considered good. The authors' details, type of study, treatment groups, sex of patients and overall survival rates (in 1, 3 and 5 years) reported in the selected articles were manually extracted and are summarized in Table 1. Finally, we individually analyzed the selected studies to determine whether VATS or OT was preferable and correlated with better outcomes.

\section{Search outcome}

Our literature search yielded 2,788 publications, 9 of which were eventually identified to be relevant to our research question (Table 1). By screening the titles of our initial 2,788 identified studies, we excluded 2,716 irrelevant studies and 54 studies that involved animal models or in vitro culture. Of the remaining 18 relevant studies, we further excluded 6 review articles, 2 scientific letters and 1 meta-analysis conducted previously by our team (Figure 1 ).

Of the 9 selected studies, Nakajima et al. (11) and Nakas et al. (16) reported no significant differences in the survival outcomes between VATS and OT patients. However, Gossot et al. (15) found that VATS correlated with improved overall survival at 1, 3 and 5 years post operation compared with OT (VATS: $87.4 \%, 70.9 \%$ and $52.5 \%$; OT: $82.3 \%, 63.6 \%$ and 34\%, respectively). Moreover, Nakajima et al. (13) and Carballo et al. (14) found that the 5 -year survival rate was better for VATS than for OT patients (Nakajima: 49.3\% vs. $39.5 \%$; Carballo: $69.6 \%$ vs. $58.8 \%$ ). Similarly, Chao et al. (17) reported that VATS had no additional negative effects on patient survival compared with OT (5 years survival rate $51 \%$ vs. $43 \%$ ).

All 9 studies provided data for the 1-, 3- and 5-year survival rates. In particular, metastases from colorectal cancer were investigated in three studies, and metastases from sarcoma were investigated in one study. The number and gender ratio of patients who underwent VATS and OT are listed in Table 1. We also summarized the key results of all selected studies. Taken together, our findings provide evidence that VATS is a safe, efficacious and promising alternative approach to OT for patients who require PM.

\section{Discussion}

The very first VATS PM was carried out in the 1990s. Under the 2014 National Comprehensive Cancer Network guidelines, VATS can be prescribed to primary lung cancer patients with no anatomical or surgical contraindications. Despite it not being a standard PM procedure, $40 \%$ of surgeons from the European Society of Thoracic Surgeons were reported to have performed VATS in 2008 (20). Due to its technical nature, intraoperative palpation, which is effective in revealing previously undiscovered metastases in $20-25 \%$ of patients $(9,21)$, is not possible in VATS. Furthermore, VATS is associated with a higher recurrence 


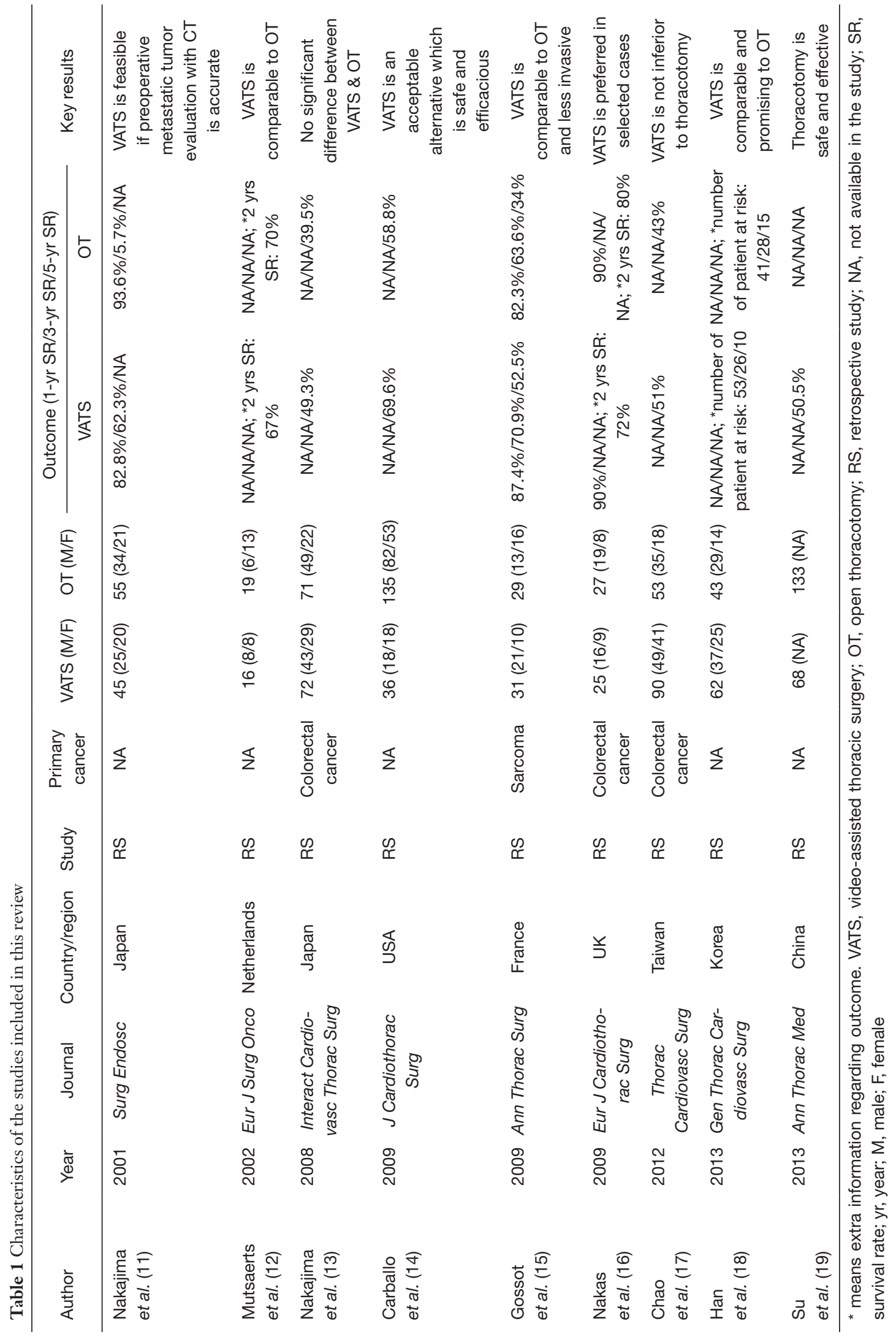




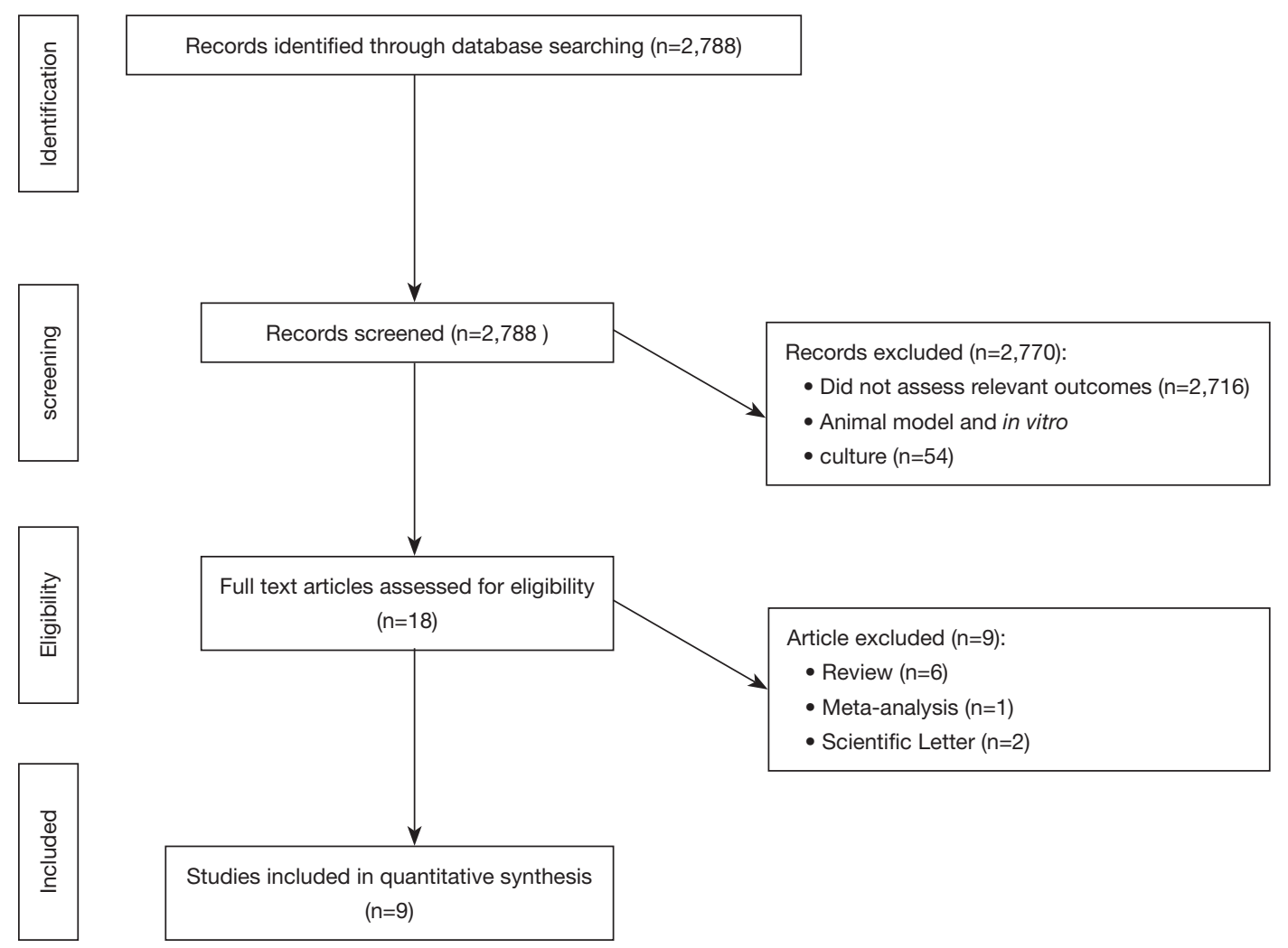

Figure 1 Flow diagram of systematic literature search results.

rate. It is therefore important to determine whether VATS may lead to worse survival outcomes in patients with pulmonary metastases compared with conventional OT (22).

In 2016, we conducted a meta-analysis and found that VATS correlated with a better overall survival rate in PM than OT (22). Furthermore, the overall survival rates of patients who underwent VATS were found to be $87.4 \%$ at 1 year and $70.9 \%$ at 3 years by Gossot et al. (15), $62.3 \%$ at 3 years by Nakajima and colleagues (11), $69.9 \%$ at 5 years by Carballo and associates (14), and $51 \%$ at 5 years by Chao and colleagues (17). Indeed, VATS was demonstrated to be an equivalent PM procedure to OT in terms of its safety and efficacy (16), and can be used as an alternative PM approach for patients with resectable pulmonary metastases $(22,23)$. Compared with OT, VATS elicits minimal surgical trauma and pain (24) and better preserves the respiratory function, which together dramatically improve patients' quality of life (25). In addition, VATS is associated with reduced hospital stay, cheaper cost, faster recovery and fewer complications (12).

In contrast, a prospective study in 1996 by McCormack et al. argued that VATS should only be used as a diagnostic tool and that classic OT still correlated with more satisfactory long-term results in lung metastases management (26). Moreover, Saisho et al. [2009] concluded that metastasectomy by VATS per se is not superior to conventional OT and must be performed by skilled surgeons (27) to ensure that all nodules are identified (28). The unique ability to perform complete palpation of the lungs remains a strong argument for open surgery $(19,29)$. Computed tomography (CT) and Positron Emission tomography (PET-CT) can determine the location and number of metastases preoperatively with moderate accuracy. However, $32.5 \%$ and $50 \%$ of nodules go undetected in PET-CT (30) and CT (31), respectively. In particular, small nodules in the lungs are often undetected by CT. While intraoperative finger palpitation can increase the detection rate of these previously unidentified nodules during conventional OT $(26,32)$, this is impossible in VATS due to the small portholes $(8,9)$. In line with this, patients who are treated using VATS experience a higher rate of recurrent pulmonary metastases (13) and are more likely to undergo additional surgeries.

There are some limitations of our study. First, it is likely that most of our included retrospective studies contained 
selection bias and other inherent limitations. Second, the lack of randomized controlled trials makes it difficult to argue for the benefits of adopting the VATS approach as the primary treatment for patients with pulmonary metastases.

In conclusion, VATS is generally a good surgical technique for treating resectable oligo metastatic lesions in the lungs. As VATS provides a minimally invasive approach, the outcomes of any further operations will also be improved. The coverage of VATS is expected to grow as surgical and imaging technologies develop. Compared with VATS, OT provides a better field of palpation for previously undetected lesions. OT also has an advantage in lung parenchyma sparing, as certain area of the lung parenchyma often needs to be resected due to angle incision in VATS. Despite that VATS is associated with many clinical benefits, more prospective studies are required to determine the indications for VATS in patients with pulmonary metastases.

\section{Acknowledgments}

Funding: None.

\section{Footnote}

Provenance and Peer Review: This article was commissioned by the Guest Editor (Khosro Hekmat) for the series "Pulmonary Metastases" published in Fournal of Thoracic Disease. The article has undergone external peer review.

Reporting Checklist: The authors have completed the Narrative Review reporting checklist. Available at: http:// dx.doi.org/10.21037/jtd-19-3958

Conflicts of Interest: The authors have completed the ICMJE uniform disclosure form (available at: http:// dx.doi.org/10.21037/jtd-19-3958). The series "Pulmonary Metastases" was commissioned by the editorial office without any funding or sponsorship. The authors have no other conflicts of interest to declare.

Ethical Statement: The authors are accountable for all aspects of this work in ensuring that questions related to the accuracy or integrity of any part of this work are appropriately investigated and resolved.

Open Access Statement: This is an Open Access article distributed in accordance with the Creative Commons Attribution-NonCommercial-NoDerivs 4.0 International
License (CC BY-NC-ND 4.0), which permits the noncommercial replication and distribution of the article with the strict proviso that no changes or edits are made and the original work is properly cited (including links to both the formal publication through the relevant DOI and the license). See: https://creativecommons.org/licenses/by-nc-nd/4.0/.

\section{References}

1. Pastorino U, Buyse M, Friedel G, et al. Long-term results of lung metastasectomy: prognostic analyses based on 5206 cases. J Thorac Cardiovasc Surg 1997;113:37-49.

2. Treasure T, Fallowfield L, Lees B. Pulmonary metastasectomy in colorectal cancer: the PulMiCC trial. J Thorac Oncol 2010;5:S203-6.

3. Rusch VW. Pulmonary metastasectomy: a moving target. J Thorac Oncol 2010;5:S130-1.

4. Moffatt SD, Mitchell JD, Whyte RI. Role of video-assisted thoracoscopic surgery and classic thoracotomy in lung cancer management. Curr Opin Pulm Med 2002;8:281-6.

5. Borasio P, Gisabella $M$, Billé A, et al. Role of surgical resection in colorectal lung metastases: analysis of 137 patients. Int J Colorectal Dis 2011;26:183-90.

6. Park JS, Kim HK, Choi YS, et al. Outcomes after repeated resection for recurrent pulmonary metastases from colorectal cancer. Ann Oncol 2010;21:1285-9.

7. Kandioler D, Krömer E, Tüchler H, et al. Long-term results after repeated surgical removal of pulmonary metastases. Ann Thorac Surg 1998;65:909-12.

8. Cerfolio RJ, Bryant AS, McCarty TP, et al. A prospective study to determine the incidence of non-imaged malignant pulmonary nodules in patients who undergo metastasectomy by thoracotomy with lung palpation. Ann Thorac Surg 2011;91:1696-700; discussion 1700-1.

9. Detterbeck FC, Grodzki T, Gleeson F, et al. Imaging requirements in the practice of pulmonary metastasectomy. J Thorac Oncol 2010;5:S134-9.

10. Jadad AR, Moore RA, Carroll D, et al. Assessing the quality of reports of randomized clinical trials: is blinding necessary? Control Clin Trials 1996;17:1-12.

11. Nakajima J, Takamoto S, Tanaka M, et al. Thoracoscopic surgery and conventional open thoracotomy in metastatic lung cancer. Surg Endosc 2001;15:849-53.

12. Mutsaerts EL, Zoetmulder FA, Meijer S, et al. Long term survival of thoracoscopic metastasectomy vs metastasectomy by thoracotomy in patients with a solitary pulmonary lesion. Eur J Surg Oncol 2002;28:864-8.

13. Nakajima J, Murakawa T, Fukami T, et al. Is thoracoscopic 
surgery justified to treat pulmonary metastases from colorectal cancer? Interact Cardiovasc Thorac Surg 2008;7:212-6; discussion 216-7.

14. Carballo M, Maish MS, Jaroszewski DE, et al. Videoassisted thoracic surgery (VATS) as a safe alternative for the resection of pulmonary metastases: a retrospective cohort study. J Cardiothorac Surg 2009;4:13.

15. Gossot D, Radu C, Girard P, et al. Resection of pulmonary metastases from sarcoma: can some patients benefit from a less invasive approach? Ann Thorac Surg 2009;87:238-43.

16. Nakas A, Klimatsidas MN, Entwisle J, et al. Video-assisted versus open pulmonary metastasectomy: the surgeon's finger or the radiologist's eye? Eur J Cardiothorac Surg 2009;36:469-74.

17. Chao YK, Chang HC, Wu YC, et al. Management of lung metastases from colorectal cancer: video-assisted thoracoscopic surgery versus thoracotomy--a case-matched study. Thorac Cardiovasc Surg 2012;60:398-404.

18. Han KN, Kang CH, Park IK, et al. Thoracoscopic resection of solitary lung metastases evaluated by using thin-section chest computed tomography: is thoracoscopic surgery still a valid option? Gen Thorac Cardiovasc Surg 2013;61:565-70.

19. Su X, Ma G, Zhang X, et al. Surgical approach and outcomes for treatment of pulmonary metastases. Ann Thorac Med 2013;8:160-4.

20. Internullo E, Cassivi SD, Van Raemdonck D, et al. Pulmonary metastasectomy: a survey of current practice amongst members of the European Society of Thoracic Surgeons. J Thorac Oncol 2008;3:1257-66.

21. Ellis MC, Hessman CJ, Weerasinghe R, et al. Comparison of pulmonary nodule detection rates between preoperative CT imaging and intraoperative lung palpation. Am J Surg 2011;201:619-22.

22. Meng D, Fu L, Wang L, et al. Video-assisted thoracoscopic surgery versus open thoracotomy in pulmonary metastasectomy: a meta-analysis of observational studies.

Cite this article as: Rusidanmu A, Chin W, Xu J, Wang L, He Z, Lv X, Hu J. Does a thoracoscopic approach provide better outcomes for pulmonary metastases? J Thorac Dis 2021;13(4):2692-2697. doi: 10.21037/jtd-19-3958
Interact Cardiovasc Thorac Surg 2016;22:200-6.

23. Dong S, Zhang L, Li W, et al. Evaluation of video-assisted thoracoscopic surgery for pulmonary metastases: a metaanalysis. PLoS One 2014;9:e85329.

24. Landreneau RJ, Hazelrigg SR, Mack MJ, et al. Postoperative pain-related morbidity: video-assisted thoracic surgery versus thoracotomy. Ann Thorac Surg 1993;56:1285-9.

25. Balduyck B, Hendriks J, Lauwers P, et al. Quality of life evolution after lung cancer surgery: a prospective study in 100 patients. Lung Cancer 2007;56:423-31.

26. McCormack PM, Bains MS, Begg CB, et al. Role of videoassisted thoracic surgery in the treatment of pulmonary metastases: results of a prospective trial. Ann Thorac Surg 1996;62:213-6; discussion 216-7.

27. Saisho S, Nakata M, Sawada S, et al. Evaluation of videoassisted thoracoscopic surgery for pulmonary metastases: 11-years of experience. Surg Endosc 2009;23:55-61.

28. Eckardt J, Licht PB. Thoracoscopic versus open pulmonary metastasectomy: a prospective, sequentially controlled study. Chest 2012;142:1598-602.

29. Eckardt J, Licht PB. Thoracoscopic or open surgery for pulmonary metastasectomy: an observer blinded study. Ann Thorac Surg 2014;98:466-9; discussion 469-70.

30. Fortes DL, Allen MS, Lowe VJ, et al. The sensitivity of 18F-fluorodeoxyglucose positron emission tomography in the evaluation of metastatic pulmonary nodules. Eur J Cardiothorac Surg 2008;34:1223-7.

31. Long H, Zheng Y, Situ D, et al. Hand-assisted thoracoscopic surgery for bilateral lung metastasectomy through sternocostal triangle access. Ann Thorac Surg 2011;91:852-8.

32. Margaritora S, Porziella V, D'Andrilli A, et al. Pulmonary metastases: can accurate radiological evaluation avoid thoracotomic approach? Eur J Cardiothorac Surg 2002;21:1111-4. 